Revista Legado de Arquitectura y Diseño ISSN: 2007-3615

ISSN: 2448-749X

legado_fad@yahoo.com.mx

Universidad Autónoma del Estado de México

México

\title{
CONSIDERACIONES ESTRATÉGICAS PARA OPTIMIZAR EL DISEÑO DE LA TRANSFERENCIA TECNOLÓGICA DENTRO DE LAS INSTITUCIONES DE EDUCACIÓN SUPERIOR
}

Benítez-Abarca, Roxana Paola; Rubio-Toledo, Miguel Ángel

CONSIDERACIONES ESTRATÉGICAS PARA OPTIMIZAR EL DISEÑO DE LA TRANSFERENCIA

TECNOLÓGICA DENTRO DE LAS INSTITUCIONES DE EDUCACIÓN SUPERIOR

Revista Legado de Arquitectura y Diseño, vol. 15, núm. 27, 2020

Universidad Autónoma del Estado de México, México

Disponible en: http://www.redalyc.org/articulo.oa?id=477963263001

Esta obra está bajo una Licencia Creative Commons Atribución-NoComercial-SinDerivar 4.0 Internacional. 
STRATEGIC CONSIDERATIONS TO OPTIMIZE THE
DESIGN OF TECHNOLOGICAL TRANSFER WITHIN
INSTITUTIONS OF HIGHER EDUCATION

Roxana Paola Benítez-Abarca arq.rpbenitez@gmail.com Universidad Autónoma de Querétaro, México

Miguel Ángel Rubio-Toledo miguelblond72@yahoo.com.mx Universidad Autónoma del Estado de México, México
Revista Legado de Arquitectura y Diseño, vol. 15 , núm. 27,2020

Universidad Autónoma del Estado de México, México

Recepción: 02 Junio 2019 Aprobación: 11 Noviembre 2019

Redalyc: http://www.redalyc.org/ articulo.oa?id $=477963263001$
Resumen: En la actualidad, el capital humano, el conocimiento y la tecnología son referentes importantes en el sector productivo. En los tres casos, son las Instituciones de Educación Superior quienes capacitan a sus estudiantes para desarrollar dichos conocimientos y tecnología una vez egresados o aún en las aulas. De tal suerte, resulta necesario que se observen los modos más adecuados para que los miembros de las Instituciones educativas se vinculen con la sociedad, ya sea en la iniciativa privada o sectores públicos. Diversos países han realizado con éxito dichas articulaciones estratégicas, no obstante, en México aún falta mucho para lograrlo. Si bien, existen estrategias institucionales que han dado excelentes resultados, tales como las incubadoras, los programas de prácticas profesionales, los incipientes departamentos de vinculación de las Instituciones Educativas, o incluso programas de las áreas científicas nacionales, los esfuerzos son incipientes. Así, el objetivo del presente trabajo es la conceptualización, discusión y consideración de diversos esfuerzos teóricos relacionados con la transferencia tecnológica, como acicate para lograr diseñar adecuadamente las estrategias en las Instituciones de Educación Superior que optimicen los esfuerzos en beneficio de las partes, es decir, la sociedad, la empresa e industria, el Estado y por supuesto la academia, con el objeto de responder a las necesidades de la población.

Palabras clave: Diseño de estrategias, Educación Superior, esquemas de vinculación, transferencia tecnológica.

Abstract: Nowadays, human capital, knowledge and technology are the most important references in the productive sector. In all three cases, it is the Higher Education Institutions that intensely prepare their students to develop such knowledge and technology once they are graduated, or even when they are still in the classrooms. In this way, it is necessary to observe the most appropriate ways for members of educational institutions to link with society, whether in the private sector or in the public sector. Already several countries have successfully made such strategic articulations, however, in Mexico there is still a long way to go. While there are already certain institutional strategies that have given excellent results, such as incubators, professional internship programs, the incipient linking departments of Educational Institutions, or even programs of the national scientific areas, the efforts are incipient. Thus, the aim of this work is the conceptualization, discussion and consideration of various theoretical efforts related to technology transfer, as an incentive to design more adequately, strategies in Higher Education Institutions that optimize efforts to benefit the 
parties, that is, Society, companies and industry, the State and of course the academy, in order to respond to the needs of the population.

Keywords: Design of strategies, Higher Education, linking scheme, technological transfer.

\section{INTRODUCCIÓN}

En diversos países se reconoce que la investigación científica y tecnológica desempeña un papel cardinal en el desarrollo de la sociedad de la información (Cumbre Mundial sobre la Sociedad de la Información, 2005). La ciencia y la tecnología son factores clave para el desarrollo de las naciones, los países altamente competitivos y desarrollados obtienen altos niveles de bienestar social debido a la capacidad de generar conocimiento e investigación, los cuales llegan a convertirse en productos y/o servicios que generan emprendimiento, más empleo y un bien social (Vega, 2012).

Estudios recientes demuestran una existente correlación entre el crecimiento económico y la capacidad que tienen los países para producir conocimiento, lo cual se traduce en desarrollo tecnológico y, a su vez, una inversión mayor en desarrollo de investigaciones y el aumento de patentes registradas. Lo anterior se observa en beneficios sociales cuando los procesos de investigación son canalizados para resolver problemas particulares de la población. Se trata de la facultad de proponer soluciones para problemas sociales mediante la innovación desde la ciencia y la tecnología.

Según Argüelles (2008), el conocimiento carga con la responsabilidad de la inversión en la tecnología y la ciencia en la mayoría de los países desarrollados, no obstante, en México resulta poco común. López (2019) señala, a modo de resumen e idea conclusiva principal del primer panel del Foro Binacional Canadá-México Ciencia, Tecnología y Desarrollo, organizado por la Universidad Nacional Autónoma de México y la Secretaría de Relaciones Exteriores, la necesaria convergencia entre la academia, la empresa, el gobierno y el pueblo, y como tarea pendiente el aprovechamiento de la innovación.

Algunas universidades mexicanas suelen firmar convenios con empresas e industrias a quienes en su momento le solicitan alguna solución específica a un problema particular. Sin embargo, los frutos obtenidos no se invierten de manera clara o contundente en la mejora de su propia infraestructura, sino es el Estado quien otorga los recursos para ello. Algo parecido, aun cuando menos grave, sucede en países más desarrollados en el ámbito tecnológico; en Estados Unidos, por ejemplo, el gasto de desarrollo tecnológico e innovación es el $2.7 \%$ del Producto Interno Bruto (PIB) y el $0.71 \%$ corresponde a la inversión pública (Trejo, 2016).

Es decir, no todos los recursos para la Investigación y Desarrollo (I+D) provienen del Estado o de las dependencias derivadas de éste, una parte importante sí emana de la iniciativa privada. Lo anterior resulta relevante, además, dada la coyuntura de las políticas de austeridad implementadas en México con la llegada del nuevo gobierno federal. Existen reducciones 
importantes a los presupuestos de las universidades públicas y a los centros de investigación, lo cual impacta por añadidura no sólo al personal de investigación, sino particularmente a la adquisición de infraestructura necesaria para desarrollar ciencia y tecnología (Álvarez-Buylla, 2018).

Es necesario entonces, observar estratégicamente la manera de beneficiarse mutuamente de los recursos que la iniciativa privada destina para la $\mathrm{I}+\mathrm{D}$, que son parte del usufructo obtenido por las mismas y se destina para el desarrollo de la empresa e industria. Ello permite, desde las Instituciones de Educación Superior (IES), quienes desarrollan el conocimiento científico y tecnológico de frontera, obtener los medios para crecer y desarrollarse de manera sostenible. Es justamente en este sentido que se implica a la Transferencia Tecnológica (TT), entendida como el proceso por el cual los conocimientos científicos y tecnológicos se transfieren de una organización a otra para desarrollar nuevas aplicaciones o en su defecto mejorar sus procesos.

Además, los desarrollos tecnológicos en el mayor de los casos ocurren en las IES. En México, dentro de las fuentes de transferencia de tecnología se encuentran los centros de investigación, laboratorios, centros tecnológicos e incluso -en menor medida- otras empresas. Este proceso implica el adquirir, ceder, compartir, licenciar, acceder a posicionar conocimiento innovador en el mercado. Así, para abonar al objetivo descrito en la introducción de este trabajo, se busca problematizar las posibilidades de la transferencia tecnológica para establecer consideraciones estratégicas en el ámbito de las IES, con el objeto de que la $\mathrm{I}+\mathrm{D}$ resulte de mayor relevancia para el sector productivo.

\section{LA TRANSFERENCIA TECNOLÓGICA (TT) Y LAS INSTITUCIONES DE EDUCACIÓN SUPERIOR (IES)}

La generación de conocimiento científico y tecnológico es el objetivo que tiene la ciencia para comprender los fenómenos presentes en diferentes contextos de realidad. En términos organizacionales, la generación de conocimiento es la habilidad de una organización para desarrollar ideas novedosas y soluciones útiles a determinados problemas (Vázquez, 2017). Entonces, el conocimiento como un proceso evolutivo, sistémico y dinámico (Nonaka, 1991), requiere transferirse por medio de estrategias e instrumentos, con el objeto de servir al ser humano, así como los demás sistemas que componen el mundo.

La tecnología es definida como el sistema de conocimientos, técnicas, habilidades, pericias y organización, utilizados para la producción y comercialización, y el uso de los bienes y servicios que satisfagan las necesidades sociales y económicas (González, 2011). Su objetivo es mejorar la calidad de vida del hombre, desarrollar su productividad, rapidez y eficacia en sus actividades diarias cotidianas,[1] para lo que sus principios son los conocimientos científicos que le permiten observar una necesidad y proponer una solución. Así, el conocimiento científico permite el desarrollo de innovaciones y nuevas tecnologías, y realimenta la información o el conocimiento. 


\section{Desde la segunda mitad del siglo $X X$, el valor socioeconómico del conocimiento ES CULTIVADO, RECONOCIDO Y ADMINISTRADO EN PAÍSES CON MAYOR ACTIVIDAD ECONÓMICA.}

Es justamente en alusión al desarrollo y transferencia de la ciencia y la tecnología que las IES suponen un interés común. La palabra transferencia indica la acción de transferir, haciendo referencia a la capacidad de transportar de un lado a otro un objeto o una cosa. En Estados Unidos el tema de la TT se aborda durante la Segunda Guerra Mundial, destacando la importancia de la investigación como fuente económica. En Latinoamérica durante la época de los años 60 surge el nuevo modelo de desarrollo económico impulsado en la Comisión Económica para América Latina (CEPAL) (Beteta, 2012).

En la Décima Cuarta Sesión de la Organización Mundial de la Propiedad Intelectual (OMPI), celebrada en Ginebra el 25 al 29 de enero de 2010, plantea la siguiente definición de transferencia de tecnología:

En el contexto de la transferencia de tecnología del sector público y de las universidades al sector privado, el término "transferencia de tecnología" se utiliza a veces en sentido más estricto: se trata de un sinónimo de "comercialización de tecnología” mediante el cual se aplican los resultados de investigaciones científicas básicas efectuadas por universidades y organismos de investigación públicos a los productos comerciales y prácticos de empresas privadas que tienen como destino el mercado (OMPI, 2010: 4).

Así, la TT trata sobre el proceso por el cual se transfieren descubrimientos científico-técnicos de una organización a otra para continuar su desarrollo tecnológico y eventualmente llevar a cabo la comercialización de nuevos productos, procesos, aplicaciones, materiales o servicios basados en los primeros. La investigación es financiada por las empresas, con el fin de hacer uso de los resultados, los cuales tienen como objetivo innovar los productos o procesos de la compañía o resolver problemas específicos del sector productor (Wright et al., 2008). Según Hassan (2015), la TT se refiere a la manera de obtener conocimientos, ideas, métodos, diseños y técnicas a fin de mejorar los procesos del país y con ello el desarrollo de las formas o los procesos de producción favoreciendo la competitividad del país.

Desde la segunda mitad del siglo XX, el valor socioeconómico del conocimiento es cultivado, reconocido y administrado en países con mayor actividad económica. En la mayoría de los países europeos, las universidades regionales negocian su presupuesto con los gobiernos y las comunidades de negocios locales sobre la base del impacto que tienen sus actividades en el crecimiento económico local (Benneworth, 2009; Heher, 2006; Clark, 1998; Lazzeretti y Tavoletti, 2005; Bird et al., 
1993). Países de otras latitudes, como Canadá, buscan también desarrollar los modelos y las políticas adecuadas para obtener los rendimientos económicos de la investigación en universidades (Langford et al., 2006, Bacchiocchi y Montobbio, 2009).

\section{Se trata de obtener resultados a partir de propuestas innovadoras PARA ALGÚN PROBLEMA VINCULADO CON ALGUNO DE LOS SECTORES QUE PRESENTAN UN PROBLEMA O UNA DIFICULTAD.}

Investigadores en el mundo han desarrollado sofisticados métodos para medir y modelar el impacto de la inversión en la ciencia y tecnología sobre la innovación y el crecimiento económico (Heher, 2006; Jones, 1995; Arechavala et al., 2010; Berman, 1990). La vinculación entre las universidades y la sociedad es una estrategia con la que algunas naciones buscan elevar la productividad de las empresas, haciéndolas de esta manera más competitiva, utilizando la investigación que se desarrolla en las IES. Se trata de obtener resultados a partir de propuestas innovadoras para algún problema vinculado con alguno de los sectores que presentan un problema o una dificultad.

Existen diversos estudios que discuten la relación de la universidad con el sector productivo, en términos de colaborar con las empresas y las administraciones públicas con la aplicación del conocimiento científicotécnico (Etzkowitz y Leydesdorff, 2000; Bozeman, 2000; Debackere, K. y Veugelers, R. 2005). Dicha relación comienza con la explotación legal y económica de los resultados de las investigaciones con la intención de soslayar los problemas sociales, y específicamente a las empresas interesadas. En las universidades los proyectos de investigación tienen la intención de solucionar problemas sociales o innovar dentro de un ámbito social o industrial.

En México, el tema de la vinculación ha sido impulsado por el gobierno, decretando algunas políticas desde lo nacional hasta lo estatal, no obstante, no se han logrado optimizar sus resultados en la mayoría de los casos. Existen algunas iniciativas con el fin de mejorar la relevancia de la educación superior para el mercado laboral emprendidas por la Secretaría de Educación Pública (SEP), el Consejo Nacional de Ciencia y Tecnología (CONACYT) o la Secretaría de Economía (SE), incluso muchas universidades han tenido sus propias iniciativas. La OECD (OECD Skills, Strategy, Diagnostic, Report: Mexico, 2017), sostiene que la educación y las competencias son pilares sobre los que México debe construir su futuro crecimiento y prosperidad.

De tal suerte, por medio de la educación superior, los estudiantes desarrollan competencias y conocimientos avanzados que complementan sus conocimientos básicos aprendidos en las anteriores etapas de estudio, 
como son los conocimientos técnicos y disciplinares. Todo este nuevo conocimiento adquirido y mejorado bajo supervisión de especialistas son los que complementan y cualifican al estudiante para confrontar la competencia laboral fuera de una IES. Así, la educación superior parece ser el punto clave para el desarrollo de competencias, conocimientos y habilidades fundamentales para el progreso económico y social de los países.

No obstante, las IES mexicanas no poseen la flexibilidad suficiente en sus programas educativos ni tampoco los programas de vinculación para adaptar sus actividades educativas e investigaciones a las necesidades actuales y emergentes de la economía mexicana (Badillo, 2015). La mayoría de las universidades no cuenta con departamentos de vinculación que funcionen como catapulta de proyectos científicos y tecnológicos, por lo cual los temas abordados en las actividades de investigación no están enfocados de manera directa en los problemas que puedan impactar a estas empresas y la sociedad en general.

Derivado de ello, los proyectos de investigación concluidos en su mayoría no ostentan una transferencia tecnológica real, sino sólo hipotética o tangencial. Las IES, entonces, carecen de la estrecha y necesaria vinculación entre universidad y empresa. Las IES más afortunadas han desarrollado estratégicamente programas vinculantes o "profesionalizantes" para paliar este problema, donde la investigación es la columna vertebral del programa de estudio, cuya intención es lograr el desarrollo de un proyecto que concluya en la creación de empresas, solucionar una problemática social o intervenir con alguna mejora en una empresa ya establecida mediante los conocimientos adquiridos en el programa académico.

\section{CONSIDERACIONES ESTRATÉGICAS PARA OPTIMIZAR EL DISEÑO DE LA TT DENTRO DE LAS IES}

Existen diversos modelos de vinculación entre academia y empresa, algunos para la $\mathrm{I}+\mathrm{D}$, otros más sobre la relación entre sectores sociales, gobierno, empresa y academia, así como otros más para la TT. A continuación, se describen brevemente[2] sólo algunos de ellos con el objeto de ilustrar los sesgos argumentativos, así como para obtener algunas de las directrices apropiadas para determinar los elementos que se pueden tomar en cuenta para un modelo de TT, es decir, las consideraciones estratégicas necesarias que se mencionan en el título de este trabajo.

Yeverino (2015) propone ciertas estrategias a través de las empresas denominadas spin off,[3] las cuales no son solamente las empresas formadas como tales, sino que pueden ser emanadas y constituidas en las mismas IES. Esto implica que los propios profesores y estudiantes sean quienes lleven a cabo los desarrollos tecnológicos y realicen la TT con beneficios para todos los involucrados. En un principio, las propias empresas y las IES obtienen provechos mutuos, pero especialmente sirven 
como caldo de cultivo de los propios estudiantes bajo el cobijo de la experiencia de los profesores.

Vázquez (2017) sugiere un enfoque sistémico para la TT, esto es, a partir de los factores sistema, estructura y estrategia; se pretende determinar la optimización del proceso mediante siete actividades, a saber: 1) Contratación de graduados e investigadores y estancias de estos en empresas; 2) Publicaciones, seminarios y conferencias; 3) Servicios de consultoría, asistencia técnica, pruebas de laboratorio, renta de equipo e instalaciones; 4) Conocimiento transmitido codificado y formal; 5) Investigación contratada y conjunta; 6) Licenciamiento y venta de patentes, y; 7) Uso de empresas spin-offs y start-ups. [4]

El modelo Bozeman (2014) permite adaptarse a las condiciones específicas de regiones donde las IES ubican elementos que promueven el desarrollo de la transferencia. Contempla cinco dimensiones, cuyas condiciones son: 1) Características de los agentes que transfieren, así como el entorno de la demanda, su potencial y la condición económica del objeto; 2) El objeto en su forma y contenido, así como las posibilidades de comercialización; 3) Los medios de transferencia, donde se incluyen los registro de propiedad industrial e intelectual; 4) Los destinatarios o usuarios, esto es, el cliente, la organización, la empresa, etc.; 5) El contexto de la demanda, relacionado con los factores del mercado en el entorno social, económico y cultural.

Talan (como se cita en Escamilla, 2012: 33), sostiene que existen principios que son el soporte de toda vinculación efectuada entre las organizaciones:

[...] la igualdad, entendida como la no subordinación de ninguna empresa a otra; la voluntad, entendida como los acuerdos de forma libre y el uso de recursos, con el fin de lograr el mayor aprovechamiento de los mismos; y la complementariedad, como una fuerza que aprovecha sus fortalezas y da cabida para el conocimiento de los puntos de oportunidad de ambas, siempre con vistas al logro de un objetivo en común (Escamilla, 2012: 33).

Algunos autores han sugerido que se vuelva más estrecha y se trabaje a manera de urdimbre la vinculación entre academia y empresa para optimizar resultados de I+D como TT, tal es el caso de Sotelo (2000), quien sostiene la necesidad de voltear a ver a los científicos y tecnólogos de las universidades, en virtud de que se trata de especialistas, no obstante, no siempre llegan al derrotero deseado dada la falta de interacción entre investigadores.

Para que la vinculación empresa-academia sea efectiva en la generación de desarrollos tecnológicos y la construcción de soluciones en la producción se requiere sistematizar e institucionalizar un modelo de vinculación de las IES, así como también rescatar, transformar, organizar y difundir el conocimiento que se genera -como parte de esta relación entre las IES y las empresas (Escamilla, 2012: 33).

Robbins (2000) sostiene la necesidad de realizar una adecuada administración y programación de los recursos, tales como los tiempos, objetivos, actividades, pero especialmente las redes de relación entre los sectores productivos y el académico. Por su parte, Sánchez (1998) 
recomienda la compatibilidad entre la oferta de servicios y productos de las universidades con la demanda de servicios y productos de la empresa e industria, y negociar los intereses para lograr los objetivos y optimizar los proyectos. De Ossa (2018), retoma la propuesta de un modelo dinámico de Arias y Aristizábal, para adaptarlo a sistemas de rehabilitación en ingeniería biomédica, el cual:

[...] se consolida como una versión mejorada del lineal, y tiene por objetivo la transferencia de conocimientos a través de su difusión, identificando una serie de aspectos que suelen pasar inadvertidos a la hora de transferir nuevos saberes, entre ellos se destaca: la comprensión intercultural, las habilidades de negociación y los incentivos para la investigación. Igualmente, ha de mencionarse el origen que tiene este modelo con el concepto de relacionamiento entre las empresas, las universidades y el Estado, que adquiere cada vez una connotación más relevante para los procesos de gestión del conocimiento (De Ossa, 2018: 85-86).

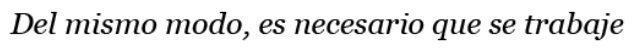 \\ A MODO DE REDES INSTITUCIONALES SISTEMATIZADAS BAJO PROGRAMAS ESTRATÉGICOS FLEXIBLES.}

Higuera (2018), finalmente, realiza una aproximación a la Educación Superior desde los sistemas de acreditación y certificación, cuyo planteamiento es justamente la articulación entre los sectores de la industria, la sociedad y el gobierno con la academia. Ello supone no sólo flexibilizar estratégicamente los programas de estudio de licenciatura y posgrado, sino principalmente su vinculación con los problemas que existen afuera de las aulas y su capacidad real de resolverlos, y que esta vinculación sea parte de los argumentos principales para otorgarle las acreditaciones y certificaciones a dichos programas de estudio como mecanismo de calidad.

Así, se pueden observar diversas posturas para la optimización estratégica de la TT en las IES, cuyo listado es meramente descriptivo y no abarca todas ellas, aun cuando permite observar ideas generales. En principio, en términos sociales, es necesario que las IES y las empresas se observen como iguales, de manera que las necesidades de las empresas sean también las necesidades de las IES, y viceversa. Es decir, lo que requiere la empresa es lo que las IES crean como objeto de su naturaleza, esto es, capital humano[5] especializado que genera conocimiento y tecnología, que es lo que a su vez requiere la empresa. En este sentido, es necesario que se observe la complementariedad simbiótica de las partes, con el fin de obtener beneficios mutuos.

En cuanto a la cultura educativa y la empresarial, es necesario observar -como sostiene Bozeman (2014) - el contexto en el que se da la TT. Se trata de realizar un diagnóstico lo más profundo posible sobre los agentes que transfieren la tecnología, el entorno de las demandas y su 
potencial económico, los medios de transferencia y sus registros legales, destinatarios intermedios (empresa) y finales (usuario) del objeto a transferir, factores del mercado y contexto espacial y temporal, así como cultural de la IES y de la empresa. Lo anterior permite conocer las condiciones en las cuales se da este proceso, y observar cualquier actividad que pueda surgir por las mismas condiciones del espacio físico y temporal de la TT.

Del mismo modo, es necesario que se trabaje a modo de redes institucionales sistematizadas bajo programas estratégicos flexibles. Es decir, la estructura en las que enmarcan los agentes académicos y empresariales es una red, regulada por los programas estratégicos que permitan -y obliguen - a las partes a desarrollar los proyectos de manera conjunta, siempre bajo la perspectiva de la innovación para la empresa y la creatividad desde la academia. Ello se logra a través de convenios firmados no sólo por las autoridades de cada organización, sino principalmente por los encargados de llevar a cabo todos los procesos y proyectos, incluyendo las organizaciones denominadas spin-offs y start-ups.

Es necesario que las IES desarrollen programas de divulgación encaminadas a procesos productivos y TT, a través de eventos como ferias, consultorías, sitios web, renta de equipos, publicaciones, entre otros, pero siempre con la idea de realizarlo hacia un sector específico, lo que coadyuva a que las empresas se acerquen a observar lo que se desarrolla como proyectos de investigación, así como sus posibles resultados. Como consecuencia, se pueden observar registros de propiedad intelectual e industrial, que incluso pueden ser objeto de venta para las empresas, pero especialmente para los propios sujetos productores de conocimiento para su desarrollo profesional y, en consecuencia, personal y social.

Las IES, finalmente, en la actual necesidad de ofrecer programas académicos de calidad en las IES, bajo las evaluaciones que les realiza la SEP, mediante el Consejo para la Acreditación de la Educación Superior (COPAES), así como el Programa Nacional de Posgrados de Calidad (PNPC), se vuelve necesario que dichos procesos de evaluación se realicen bajo una estricta observancia de la resolución de las necesidades sociales, entre las que se encuentran las empresariales, con el objeto de que la educación superior se vuelva en realidad un agente de cambio en la realidad de la población. Es necesario señalar que, el papel de las IES es la formación de personas para su desarrollo en todos los niveles, incluyendo el laboral.

\section{CONCLUSIONES}

Las universidades han realizado esfuerzos cada vez más intensos en la investigación, considerando que los resultados que se obtienen son aplicados en la industria y la sociedad. Esta acción se considera uno de los pilares básicos de la educación superior de calidad, con el objetivo de complementar las actividades de investigación y docencia principalmente (Caldera y Debande, 2010). Las IES pueden contribuir en el desarrollo social y económico de una región, para ello es necesario que estas 
instituciones implementen unidades de vinculación bajo los parámetros de la TT. Además, deben considerar una reglamentación interna la que defina cómo administrar los recursos financieros, la repartición en la institución y la protección intelectual de los conocimientos (Solleiro, 1995).

Así, la Educación Superior es esencial para que una sociedad prospere. No obstante, a pesar de que la mayoría de las universidades mexicanas cuentan con investigadores y estudiantes que crean y almacenan conocimiento de manera tecnológica, el mayor número de los casos no llegan a transferirse, mucho menos a ser aplicados dentro de la sociedad, incluyendo la empresa e industria, por la falta de entendimiento, vinculación y contacto entre investigadores y empresarios. Entonces, resulta evidente la necesidad de la vinculación entre las IES y las empresas, desarrollando estrategias suficientemente profundas y amplias para justificar la sistematización e institucionalización de dicho proceso.

En tal virtud, podemos considerar que contar con un modelo que tenga la facilidad de adaptarse a las condiciones particulares de contexto, así como la creación de redes, pueden lograr el incremento de la transferencia tecnológica con resultados exitosos en la vinculación con la industria. Lo anterior implica la creación de modelos de vinculación dirigidos a las IES, donde se justifique la necesidad de considerar departamento especializados que lleven a cabo las funciones de TT, así como determinar un marco jurídico que defina y regule dichas actividades. Se espera entonces, que la puesta en marcha de dicho manual, en primera instancia, logre una vinculación estrecha entre universidad e industria, así también se promueva y aumenten las redes para el desarrollo de las transferencias con los sectores productivos, con el objeto de lograr beneficios económicos y sociales para los involucrados en el proceso.

\section{FUENTES DE CONSULTA}

Álvarez-Buylla, R. M. E. (2018), Plan de reestructuración estratégica del Conacyt para adecuarse al Proyecto Alternativo de Nación (2018-2024) presentado por Morena, Gobierno de la República Mexicana, Ciudad de México. Disponible en http://www.smcf.org.mx/avisos/2018/planconacyt-ciencia-comprometida-con-la-sociedad.pdf, consultado el 22 de mayo de 2019.

Arechavala, R. (2010), "Innovación educativa, ¿en las universidades?", Ide@s CONCYTEG, vol. 5, núm. 61. Disponible en http:// octi.guanajuato.gob.mx/octigto, consultado el 18 de mayo de 2019.

Argüelles V. M. y Benavides G. C. (2008), "Conocimiento y crecimiento económico: una estrategia para los países en vías de desarrollo”, Revista de Economía Mundial, vol. 18, pp. 65-77.

Bacchiocchi, E. y Montobbio, F. (2009), “Knowledge diffusion from university and public research. A comparison between US, Japan and Europe using patent citations", The Journal of Technology Transfer, vol. 34, núm. 2, pp. 169-181.

Badillo Vega, R., Galan-Muros, V., Baaken, T., Raesfeld, L. (2015), Change to Success: Case Studies of Latin American Universities on Solutions 
for Promoting Innovation in Knowledge and Technology Transfer, Waxmann, Muenster/New York.

Benneworth, P., Coenen, L., Moodysson, J., Asheim, B. (2009), "Exploring the Multiple Roles of Lund University in Strengthening Scania's Regional Innovation System: Towards Institutional Learning?", European Planning Studies, vol. 27, núm. 11, p. 1645.

Berman, E. M. (1990), "The economic impact of industry-funded university R\&D”, Research Policy, vol. 19, núm. 4, pp. 349-355.

Beteta, H. y M., Juan C. (2012), "El desarrollo en las ideas de la CEPAL", Economía UNAM, vol. 9, núm. 27, pp. 76-90. Disponible en http://www.scielo.org.mx/scielo.php? script=sci_arttext\&pid=S1665-952X2012000300004\&lng=es\&tlng=es, consultado el 7 de abril de 2019.

Bird, B., Hayward, D., Allen, D. (1993), "Conflicts in the Commercializationof Knowledge: Perspectives from Science and Entrepreneurship",Entrepreneur-ship Theory and Practice, vol. 27, núm. 4, pp. 57-77.

Bozeman, B. (2000), "Technology transfer and public policy: a review of research and theory", Research Policy, vol. 29, núm. 4-5, pp. 627-655.

Bozeman, B., Rimes, H., Youtie, J. (2014), "The envolving stateof- the-art in technology transfer research: Revisiting the contingent effectiveness model”, Research Policy, vol. 44, núm. 1. pp. 34-49.

Caldera, A. and Debande, O. (2010), "Performance of Spanish universities in technology transfer:an empirical analysis”, Research Policy, vol. 39, núm. 9, pp. 1160-1173.

Clark, B. (1998), "The entrepreneurial university: Demand and response", Tertiary Education and Management, vol. 4, núm. 1, pp. 5-16.

Cumbre Mundial sobre la Sociedad de la Información (2005), Declaración de principios: construir la sociedad de la información: un desafío global para el nuevo milenio. [S.1.: Internacional Telecommunication Union]. Disponible en http://www.itu.int/wsis/docs/geneva/official/ dop-es.html, consultado el 8 de noviembre de 2019.

Debackere, K., Veugelers, R. (2005), “The role of academic technology transfer organizations in improving industry science links", Research Policy, vol. 34, núm. 3, pp. 321-342.

De Ossa, María T., Londoño, E., Valencia, A. (2018), "Modelo de Transferencia Tecnológica desde la ingeniería biomédica: un estudio de caso", Informacio\#n Tecnológica, vol. 29, núm. 1, pp. 83-90. Disponible en http://dx.doi.org/10.4067/S0718-07642018000100083, consultado el 16 de mayo de 2019.

Escamilla, S., Clara y María Teresa, G. M. (2012), "Modelo de vinculación entre las Instituciones de Educación Superior y las empresas: gestión del conocimiento", Acta Universitaria, vol. 22, núm. 2, pp. 32-40. Universidad de Guanajuato, México. Disponible en http:// www.redalyc.org/articulo.oa?id=41623191005, consultado el 21 de abril de 2019.

Etzkowitz, H. y Loet Leydesdorff (2000), "The dynamics of innovation: from National Systems and Mode 2 to a Triple Helix of university-industrygovernment relations", Research Policy, vol. 29, pp. 109-123. 
González, J. (2011), Manual de transferencia de tecnología y conocimiento. Disponible en http://www.gonzalezsbater.com/blog/2010/12/08/ manual-de-transferencia-de-tecnologia-y-conocimiento-2-edición, consultado el 2 de mayo de 2019.

Hassan, A., J. M. y Menshawi, K. (2015), "International Technology Transfer Models: A Comparison Study", Journal of Theoretical \& Applied Information Technology, vol. 78, núm.1, pp. 95-108.

Heher, A. (2006), "Return on Investment in Innovation: Implications for Institutions and National Agencies", The Journal of Technology Transferal, vol. 4, pp. 403-414.

Higuera Z. A. y Rivera G. E. (2018), "Método de la Tetra Hélice: Evaluación de Procesos de Acreditación en Diseño para Instituciones de Educación Superior en México", Actas de diseño, vol. 13, núm. 25, pp. 213-221. Disponible en https://fido.palermo.edu/servicios_dyc/publicacionesdc/ vista/detalle_articulo.php?id_libro=6898id_articulo=14445, consultado el 21 de mayo de 2019.

Jones, C. I. (1995), "R\&D-Based Models of Economic Growth", The Journal of Political Economy, vol. 103, núm. 4, pp. 759-784.

Langford, C., Hall, J., Josty, P., Matos, J., Jacobson, A. (2006), "Indicators and outcomes of Canadian university research: Proxies becoming goals?", Research Policy, vol. 35, núm. 10, pp. 1586-1598.

Lazzeretti, L. y Tavoletti, E. (2005), "Higher Education Excellence and Local Economic Development: The Case of the Entrepreneurial University of Twente”, European Planning Studies, vol. 13, núm. 3, pp. 475.

López, P. (2019), "Necesaria, la convergencia de academia, empresa, gobierno y pueblo", Gazeta Digital UNAM. 20 de mayo de 2019. Disponible en http://www.gaceta.unam.mx/necesaria-la-convergencia-de-academiaempresa-gobierno-ypueblo/?

fbclid=IwAR0yYtc0mQNdMmTEnLFWPdTU5y4stbdZWCh2NQ_...1/3, consultado el 21 de mayo de 2019.

Nonaka, I. (1991), "The knowledge-creating company", Harvard Business Review 69, pp. 96-104.

OECD (2017), "OECD Skills Strategy Diagnostic Report: Mexico 2017”, OECD Skills Studies. Disponible en http:// dx.doi.org/10.1787/9789264287679-en, consultado el 22 de abril de 2019.

Organización Mundial de la Propiedad Intelectual (OMPI) (2010), "Décima Cuarta Sesión celebrada en Ginebra del 25 al 29 de enero", Comité Permanente sobre el Derecho de Patentes, Transferencia Tecnológica.

Robbins, S., D., D. (2000), Fundamentos de Administración, Pearson Educación, México.

Sánchez Daza, G., López Leyva, S., Corona Treviño, L., Martínez de Ita, M., Riszard Rózga, L. (1998), La vinculación e investigación tecnológica: un enfoque regional. Universidad Autónoma de Puebla-Universidad de Occidente, Sinaloa, p. 50.

Solleiro, J. (1995), "Gestión de la vinculación universidad-sector productivo", Nuevos paradigmas de la vinculación universidad-industria, Universidad de Sonora, México.

Sotelo, J. A. (2000), Regional Development Models, Oxford University Press, EUA. 
Trejo P., P. (2016), Reflexiones desde la banca, Lagaras de México, México.

Vázquez G., E. R. (2017), “Transferencia del conocimiento y tecnología en universidades", Iztapalapa Revista de Ciencias Sociales y Humanidades, vol. 83, núm. 38, pp. 75-95. DOI: http://dx.doi.org/10.28928/ revistaiztapalapa/832017/atc3/vazquezgonzalezer, consultado el 22 de octubre de 2019.

Vega, M., A. (2012), "Aspectos y avances en ciencia, tecnología e innovación”, Polis (Santiago), vol. 11, núm. 33, pp. 451-470, Disponible en https:// dx.doi.org/10.4067/S0718-65682012000300022, consultado el 8 de noviembre de 2019.

Wright, M., Clarysse, B., Lockett, A., Knockaert, M. (2008), "Mid-range universities' linkages with industry: Knowledge types and the role of intermediaries", Research Pollicy, vol. 37, núm. 8, pp. 1205-1223.

Yeverino J., J. A. (2015), La transferencia tecnológica universitaria en México: un análisis de sus determinantes y sus resultados. Tesis doctoral. Universidad Complutense de Madrid.

\section{Notas}

[1] Las actividades diarias cotidianas se refieren a lo que realiza un sujeto, por ejemplo, en cuanto a higiene personal (regaderas con mecanismos de ahorro y eficiencia de uso de agua), transporte (uso de vehículos con mayor seguridad y rapidez), trabajo (sistemas electrónicos de uso de datos más exactos), entre otros.

[2] Se ofrece una disculpa por la drástica reducción de los argumentos que se presentan en los textos originales, y se invita a los lectores, en caso de que se requiera mayor información, a referirse a dichos documentos citados en este trabajo.

[3] Empresas de base tecnológica que se vuelven negocios en sí mismos, mediante la gestión de un nuevo modelo de negocio flexible.

[4] Las empresas start-ups son similares en funcionamiento a las spin-off, sin embargo, no ostentan algún tipo de contrato de propiedad intelectual con la institución.

[5] Es necesario aclarar, que en sentido estricto "Capital humano" se refiere al potencial de la producción, distribución y consumo de conocimientos generados por el ser humano, lo que le otorga la condición de capital -desde la economía epistémica- realizado por personas, sin que ello implique cosificar al hombre en su posibilidad intelectual. De tal suerte, una parte de la naturaleza de las IES es capacitar sujetos para el desarrollo de posibilidades laborales, aun cuando no se solsayan las otras necesidades de enseñanza de las IES para que los egresados produzcan bienestar y conciencia para sí y para las comunidades desde otras áreas de desarrollo humano. 